\title{
IS AUTONOMIC DYSFUNCTION INTRICATELY ASSOCIATED WITH SOMATOFORM DISORDERS? AN ANALYTICAL STUDY IN KOLKATA
}

\author{
Krishna Laskar ${ }^{1}$, Ashmita Sengupta ${ }^{2}$
}

1 Demonstrator, Department of Physiology, Diamond Harbour Government Medical College and Hospital, Kolkata, West Bengal, India. ${ }^{2}$ Assistant Professor, Department of Physiology, Midnapore Medical College and Hospital, Medinipur, West Bengal, India.

ABSTRACT
BACKGROUND
Somatoform disorder is nowadays considered as the most frequent psychiatric diagnosis in general practice and is diagnosed in
$16.1 \%$ of consecutive consulting patients. Physiological disturbances are so common in somatization that it can easily maintain the
body focus, and symptom misinterpretations of a person having somatoform dysfunction. The interaction between 'psyche' and
"soma" happens through a complex network of feed-back and modulation among the central and autonomic nervous system. We
wanted to assess the status of autonomic function as per existing standard (Ewing's) test protocol among study population and
evaluate the association between autonomic functions and somatoform disorders.

\section{METHODS}

A cross-sectional, analytical study was conducted using five non-invasive tests as per existing Ewing's test protocol among fifty newly diagnosed somatoform disorder patients of 18-40 years age group of both sexes who had been attending psychiatry outpatient department along with age and sex matched healthy subjects.

\section{RESULTS}

There was significant difference in 30:15 $(\mathrm{p}=0.02)$, VR $(\mathrm{p}=0.04)$ responses between cases \& control groups indicating reduced parasympathetic activity and comparing study subjects with control subjects no significant difference in blood pressure response to standing. IHG test response was found to exist indicating no change in sympathetic activity among somatoform disorder patients compared to control subjects.

\section{CONCLUSIONS}

Reduced parasympathetic activity and no sympathetic dysregulation were present among patients of somatoform disorders and this should be taken care of as reduced parasympathetic activity might cause cardiovascular disturbances.

HOW TO CITE THIS ARTICLE: Laskar K, Sengupta A. Is autonomic dysfunction intricately associated with somatoform disorders? an analytical study in Kolkata. J. Evolution Med. Dent. Sci. 2019;8(13):1069-1073, DOI: 10.14260/jemds/2019/236

\section{BACKGROUND}

Somatoform disorder (SD) is the presentation of functional complaints as organic or physical. Here anxiety arises when physical sensations are misinterpreted as evidence of organic pathology and physiological symptoms associated with anxiety again fuel the body focused attention. ${ }^{1}$ Somatoform disorder is nowadays considered as the most frequent psychiatric diagnosis in general practice and is diagnosed in $16.1 \%$ of consecutive consulting patients. ${ }^{2}$ The interaction between 'psyche' and "soma" are well known. This interaction happens through a complex network of feed-back and modulation among the central and autonomic nervous system, the endocrine system, immune system and the stress system. ${ }^{3}$

Physiological disturbances are so common in somatization that it can easily maintain the body focus and symptom misinterpretations of a person having somatoform dysfunction. ${ }^{4}$

'Financial or Other Competing Interest': None.

Submission 08-02-2019, Peer Review 13-03-2019,

Acceptance 20-03-2019, Published 01-04-2019.

Corresponding Author:

Dr. Ashmita Sengupta,

39 RBC Road, Central Enclave,

Flat No. 4A, Kolkata-700028,

West Bengal, India.

E-mail: krishban69@gmail.com

DOI: $10.14260 /$ jemds $/ 2019 / 236$
A recent model by Brown (2004) addresses on this issue by showing how physical symptoms can arise and the autonomic control of action is over-determined by typical presentation of illness. ${ }^{5}$

Disorders of autonomic nervous system play a crucial role in the pathogenesis and clinical course of many diseases. Many methods evaluating the autonomic nervous system have been described. The availability of non-invasive techniques like monitoring subtle changes in heart rate and blood pressure are now replacing earlier painful techniques. The heart rate and blood pressure can be mapped during rest as well as to certain provocative manoeuvres. Given the complexity of the autonomic nervous system there is no single test that precisely reflects function of a specific branch of this system.

As such there is no definite study to determine whether the persons misinterpreting their normal bodily sensations, do really have any physiological difference in autonomic functions. Some tests to assess autonomic function had been chosen as per existing Ewing's test protocol6. These tests were performed among newly diagnosed somatoform disorder patients of 18- 40 years age group of both sexes who had been attending psychiatry OPD along with age and sex matched healthy subjects.

With this background, the present study was conducted among newly diagnosed patients of somatoform disorders and their age and sex matched controls in a government teaching hospital in Kolkata with the following Objectives: 
1. To assess the status of autonomic function as per existing standard (Ewing's) test protocol among study population.

2. To find out the association between autonomic functions and somatoform disorders.

\section{METHODS}

\section{Study Type, Design and Population}

A cross-sectional, analytical study was conducted during March 2015- February 2016 at Department of Physiology and Psychiatry out-patient department (OPD) of the teaching hospital. Study group comprised of all the patients attending Psychiatry OPD of the hospital for the first time and diagnosed as Somatoform disorders as per diagnostic guidelines of ICD $10^{7}$ during the study period. Their age and sex matched accompanying person without any psychiatric morbidity were selected as a control group. The ratio of study and control group was 1:1.

\section{Sample Size and Sampling Design}

In this study consecutive sampling method was used. The Out-patient Department (OPD) day was selected randomly per week. On that particular day during the study period all newly diagnosed cases of somatoform disorder within 18 40 years age group were recruited from.

For selection of controls, among the companion of the patients age and sex match similar number of subjects were selected randomly and recruited after screening using GHQ288.

\section{Exclusion Criteria}

Individuals having cardiac disease, hypertension, diabetes mellitus, neurological disease, retinopathy, nephropathy, autoimmune diseases, any other acute or chronic systemic disease(s) or associated factor(s) that may affect the autonomic reflexes were not included in the study or control group.

\section{Methods of Data Collection}

Clinical history was taken from each individual and general examination was performed by the competent Psychiatrists. Autonomic function tests were performed using standard battery of tests in laboratory setting according to the protocol of Ewing and Clarke6. Pre-test instructions were given to avoid consumption of any medications that may alter the autonomic function 48 hours prior to the test. No study subjects were allowed to consume cigarette, nicotine, coffee, food or medicines from two hours prior to the test. ${ }^{6}$ The subjects were asked to wear loose gowns, and to remove tight under clothing, metallic objects like rings, watches etc.

The subjects were asked to take rest for 15 minutes in supine position just before the commencement of the tests. The resting time after each test was 5 to 10 minutes. ${ }^{6}$ The tests were performed in an equable environment with room temperature ranging from $18^{\circ}-28^{\circ} \mathrm{C}$. A basal recording of the resting heart rate (RHR) by using Polyrite-D, and blood pressure for screening and drawing a baseline was taken first, followed by the autonomic function tests. The following test parameters were assessed in the specific sequence as given below.

\section{Autonomic Function Tests for Parasympathetic Activity}

1. Heart rate response to postural change (30:15).

2. Heart rate variation during Deep breathing (HRDB) or Deep Breath Difference (DBD).

3. Heart rate response to Valsalva manoeuvre (Valsalva Ratio or VR).

\section{Autonomic Function Tests for Sympathetic Activity \\ 1. Blood pressure response to standing (Orthostatic test). \\ 2. Blood pressure response to sustained isometric hand- grip (IHG).}
Study Tools and Techniques
A. Case Record sheet
B. General Health Questionnaire-28
C. Polyrite D (Recorder and Medicare Systems Pvt. Ltd.)

\section{(A) Tests for Autonomic Function6}

\section{Heart Rate Response to Postural Change (30:15)}

After a complete rest of 15 minutes in supine position the ECG recording was started, and the subject assumed erect posture from the supine position as quickly as possible (within 3 seconds) with continuous ECG recording for 30 seconds or more in erect posture. The ratio of the longest RR interval around $30^{\text {th }}$ beat after standing to the shortest RR interval around $15^{\text {th }}$ beat after standing was calculated for result of 30:15.

\section{Heart Rate Variation During Deep Breathing (HRDB) or Deep Breath Difference (DBD)}

The patient was instructed to take deep inspiration over 5 secs and followed by expiration over next 5 secs completing one respiratory cycle and six cycles were repeated. The difference of the heart rate between the maximum in the inspiratory cycle (I) and the minimum in the expiratory cycles (E) was calculated and used as the result.

\section{Heart Rate Response to Valsalva Manoeuvre}

A mercurial sphygmomanometer was improvised by modifying with a mouthpiece and a body tube of a $10 \mathrm{ml}$ disposable hypodermic syringe in place of the air pump, and connected directly to the tube leading to mercury bulb6. A small leak in the mouth piece was allowed. ${ }^{9}$

The subject was instructed to exhale forcefully through the mouth piece of the modified mercurial sphygmomanometer and to maintain pressure in the manometer up to $40 \mathrm{mmHg}$ for 15 seconds. ECG recording was taken during the manoeuvre and continued for about 30 seconds after the performance. Nasal clip was used to stop nasal breathing of the subject during this manoeuvre. The ratio of the longest RR interval after blowing to the shortest RR interval during blowing was calculated. The highest ratio of the three manoeuvres was used as the result of Valsalva ratio (VR).

\section{Blood Pressure Response to Standing (Orthostatic Test)}

Resting blood pressure was recorded. Then the patient was asked to stand up and the blood pressure was recorded immediately (' 0 ' minute). The difference between the systolic blood pressure (SBP) while lying down and the SBP while the subject stood, was calculated. The fall in systolic blood pressure was used as the result of orthostatic test. 
Blood Pressure Response to Sustained Isometric HandGrip (IHG)

In sitting position, the subject was asked to press the hand grip dynamometer at $30 \%$ of maximum voluntary contraction for two minutes. Blood pressure was measured before and after contraction at two minutes in contralateral arm. Difference in resting diastolic blood pressure (DBP) before hand-grip and highest DBP during hand-grip was noted.

\section{Statistical Analysis}

All the collected data were at first entered a Microsoft Excel spreadsheet and were checked for consistency. Percentage mean ( \pm standard deviation) of the data was calculated. After that association between variables was tested with Pearson's chi-square test and Fischer's exact test where Cochrane criteria was not fulfilled.

\begin{tabular}{|c|c|c|c|c|}
\hline Tests & Parameters & Normal & Borderline & Abnormal \\
\hline $\begin{array}{c}\text { Heart Rate } \\
\text { Response to } \\
\text { Standing }\end{array}$ & $30: 15$ & $\geq 1.04$ & $1.01-1.03$ & $\leq 1.00$ \\
\hline $\begin{array}{c}\text { Heart Rate } \\
\text { Response to } \\
\text { Deep } \\
\text { Breathing }\end{array}$ & $\begin{array}{c}\text { Maximum } \\
\text { Minus } \\
\text { Minimum } \\
\text { Heart Rate } \\
\text { (E-I) }\end{array}$ & $\geq 15$ & $11-14$ & $\leq 10$ \\
\hline $\begin{array}{c}\text { Heart Rate } \\
\text { Response to } \\
\text { Valsalva } \\
\text { Manoeuvre }\end{array}$ & $\begin{array}{c}\text { Valsalva Ratio } \\
\text { (VR) }\end{array}$ & $\geq 1.21$ & $1.11-1.20$ & $\leq 1.10$ \\
\hline $\begin{array}{c}\text { Blood } \\
\text { Pressure } \\
\text { Response to } \\
\text { Standing }\end{array}$ & $\begin{array}{c}\text { Fall in SBP } \\
\text { (mmHg) }\end{array}$ & $\leq 10$ & $11-29$ & $\geq 30$ \\
\hline $\begin{array}{c}\text { Blood } \\
\text { Pressure } \\
\text { Response to } \\
\text { IHG }\end{array}$ & $\begin{array}{c}\text { Rise in DBP } \\
\text { (mmHg) }\end{array}$ & $\geq 16$ & $11-15$ & $\leq 10$ \\
\hline Normative Values of Cardiovascular Autonomic Function6 \\
\hline
\end{tabular}

\section{Ethical Consideration}

The study was commenced after receiving clearance from Institutional Ethics Committee. Written informed consent was obtained from each study subject.

\section{Additional Resources}

Not applicable.

\section{RESULTS}

The current study is an analytical observational study among fifty subjects who have been newly diagnosed as case of somatoform disorder by psychiatrists of the hospital and fifty age and sex matched healthy adults to assess and compare the cardiovascular autonomic functions. Over the study period, a total number of sixty-one newly diagnosed somatoform disorder patients had been referred from Psychiatry OPD, who gave informed consent to participate in the study. Due to presence of one or more co-morbidities or inability to perform the tests, 11 study subjects were excluded from the study in the beginning. The final size of study group was fifty. Equal number of age \& sex matched persons fulfilling the selection criteria were included in the control group.
Among the case $80 \%$ were female and among control $66 \%$ were female. As per the test result there was no significant difference (Pearson Chi square test: Value $=2.468$, $\mathrm{p}=0.113$ ) between case and controls regarding gender. According to table 1; significant difference was present in 30:15 ( $\mathrm{p}=0.02)$, VR $(\mathrm{p}=0.04)$ responses between somatoform disorder patients \& control groups and there was no significant difference in heart rate variation to deep breathing, blood pressure response to standing or IHG test response of case and control groups.

Table 2 showed that there is significant difference in mean score of 30:15 ( $p=0.003)$, VR ( $p=0.01)$ of parasympathetic function and no significant difference in sympathetic test results between cases \& control groups.

\begin{tabular}{|c|c|c|c|c|}
\hline $\begin{array}{l}\text { Study } \\
\text { Variables of } \\
\text { Autonomic } \\
\text { Function }\end{array}$ & $\begin{array}{l}\text { Variable } \\
\text { Categories }\end{array}$ & $\begin{array}{c}\text { Cases } \\
(\%)\end{array}$ & $\begin{array}{c}\text { Control } \\
\text { (\%) }\end{array}$ & $\begin{array}{c}\chi^{2} / \\
\text { Fisher's } \\
\text { Exact Test } \\
\text { (p value) }\end{array}$ \\
\hline \multirow{3}{*}{$30: 15$} & Abnormal & $2(4.0)$ & 0 & \multirow{3}{*}{$\begin{array}{c}5.26 \\
(0.02)\end{array}$} \\
\hline & Borderline & $3(6.0)$ & $0(0.0)$ & \\
\hline & Normal & $45(90.0)$ & $50(100.0)$ & \\
\hline \multirow{2}{*}{ DBD } & Abnormal & $1(2.0)$ & $0(0.0)$ & \multirow{2}{*}{$\begin{array}{c}1.00^{*} \\
(0.317)\end{array}$} \\
\hline & Normal & $49(98.0)$ & $50(100.0)$ & \\
\hline \multirow{2}{*}{ VR } & Borderline & $4(10.0)$ & $0(0.0)$ & \multirow{2}{*}{$\begin{array}{c}4.125^{*} \\
(0.042)\end{array}$} \\
\hline & Normal & $46(92.0)$ & $50(100.0)$ & \\
\hline \multirow{2}{*}{$\begin{array}{l}\text { Blood Pressure } \\
\text { Response to } \\
\text { Standing }\end{array}$} & Normal & $37(74.0)$ & $30(60.0)$ & \multirow{2}{*}{$\begin{array}{c}1.654 \\
(0.198)\end{array}$} \\
\hline & Borderline & $13(26.0)$ & $20(40.0)$ & \\
\hline \multirow{3}{*}{ IHG Test } & Normal & $25(50)$ & $30(60.0)$ & \multirow{3}{*}{$\begin{array}{l}1.295 \\
(0.52)\end{array}$} \\
\hline & Borderline & $13(26.0)$ & $12(24.0)$ & \\
\hline & Abnormal & $12(24.0)$ & $8(16.0)$ & \\
\hline \multicolumn{5}{|c|}{$\begin{array}{c}\text { Table 1. Comparison of Different Categories of Study } \\
\text { Variables of Autonomic Functions Between Cases \& } \\
\text { Control Groups }\end{array}$} \\
\hline \multicolumn{5}{|c|}{ * Fisher's Exact Test is done } \\
\hline
\end{tabular}

\begin{tabular}{|c|c|c|c|}
\hline $\begin{array}{c}\text { Study } \\
\text { Variables of } \\
\text { Autonomic } \\
\text { Function }\end{array}$ & $\begin{array}{c}\text { Cases } \\
\text { Score } \\
\text { (Mean } \pm \text { SD) }\end{array}$ & $\begin{array}{c}\text { Controls } \\
\text { Score } \\
\text { (Mean } \pm \text { SD) }\end{array}$ & $\begin{array}{c}\text { Independent- } \\
\text { Sample t Test } \\
\text { (p Value) }\end{array}$ \\
\hline $30: 15$ & $1.19 \pm 0.15$ & $1.28 \pm 0.11$ & $-3.045(0.003)$ \\
\hline DBD - & $32.10 \pm 18.84$ & $35.72 \pm 11.38$ & $-1.163(0.248)$ \\
\hline VR & $1.66 \pm 0.31$ & $1.84 \pm 0.39$ & $-2.523(0.013)$ \\
\hline $\begin{array}{c}\text { Orthostatic } \\
\text { Test - Fall in } \\
\text { SBP } \\
\text { (mmHg) }\end{array}$ & $8.96 \pm 5.42$ & $10.28 \pm 5.51$ & $-1.207(0.23)$ \\
\hline $\begin{array}{c}\text { IHG Test- Rise } \\
\text { in DBP } \\
\text { (mmHg) }\end{array}$ & $15.36 \pm 8.27$ & $16.56 \pm 8.31$ & $-0.724(0.471)$ \\
\hline $\begin{array}{c}\text { Table 2. Comparison of Mean Score of Various Study } \\
\text { Variables of Autonomic Functions Between Cases and } \\
\text { Control Groups }\end{array}$ \\
\hline \multicolumn{4}{|c}{} \\
\hline
\end{tabular}

\section{DISCUSSION}

To the best of our knowledge there is very limited information regarding cardiovascular autonomic function test outcome in somatoform disorder patients. Possibly this is the first study where autonomic function tests using Ewing's protocol has been applied among somatoform disorder patients.

In this study the somatoform disorder patients showed reduced parasympathetic activity which may be explained by Porge's Polyvagal theory. According to this theory the vagus 
nerve has a modulating or regulating role and connects to various brain regions and serves as a brake or modulator. Conditions of extreme stress break down this highly sensitive means for stabilizing the human organism. This thereby causes decreased parasympathetic activity in somatoform disorder patients 10 .

The study subjects consisted of different age groups ranging from $18-40$ years with the mean age $29.04 \pm 6.8$ years. Different studies like that of Deveci A. et al in Turkey (2007), showed the peak incidence in the mid to late thirties ${ }^{11}$. This may be due to the fact that the symptom onset of somatoform disorder usually occurs in the adolescence or before and at least some of the symptoms must be developed before the age of thirty ${ }^{12}$. No significant difference $(p>0.05)$ was found between case and control groups regarding age and sex distribution in present study. Therefore, it can be concluded that case and control groups were age and sex matched.

The autonomic nervous system includes a neural mechanism in which most of its functions are carried out involuntarily. Both parasympathetic and sympathetic nervous system control heart rate, blood pressure and maintain the balance. Resting heart rate can be used to assess both SNS and PNS reactivity ${ }^{13}$. In the present study, mean resting heart rate was higher in somatoform disorder patients $(85.98 \pm 10.69)$ than control subjects $(72.54 \pm 7.15)$. Compared to control a significant difference $(p=0.006)$ of resting heart rate was also found among somatoform disorder patients. This difference in resting heart rate between somatoform disorder patients and control group was clinically significant. This may be explained by reduced parasympathetic activity. A study by Filiz I. et al in 2015 showed that mean heart rate was $86 \pm 13$ beats per minute among conversion disorder patients which is a sub variant of somatoform disorder 14,15. In the present study resting systolic and diastolic blood pressure was in the normal range in both case and control groups and no significant difference were also seen among case and control subjects. This may presumably because of multiple blood pressure regulatory systems running in parallel and autonomic disturbances might have been counterbalanced by other regulatory mechanisms.

In the present study majority of subjects (90\%) with somatoform disorder had normal heart rate response (30: 15 ) to postural change and $6 \%$ and $4 \%$ had borderline and abnormal responses respectively and also there was a significant difference $(p=0.02)$ in 30: 15 score in case and control subjects. The mean score of 30:15 response between somatoform disorder patients and healthy adults had significant difference $(\mathrm{p}=0.003)$. In case of heart rate response to Valsalva manoeuvre (VR) $92 \%$ of somatoform disorder patients had normal Valsalva ratio and significant difference $(p=0.01)$ of mean score of VR was found between somatoform disorder patient and control group. Both the results indicate reduced parasympathetic activity in somatoform disorder patients. In a different study, Viehof Z. et al (2016) found reduced heart rate variability among somatoform disorder patients ${ }^{16}$. Heart rate variability means beat to beat variation in length of time and it is a measure of parasympathetic activity. Reduced heart rate variability means para-sympathetic disturbances. Majority of patients (98\%) showed normal heart rate response to deep breathing and while comparing the mean score of DBD of somatoform disorder patients with control subjects no significant difference $(p=0.25)$ was found. In the present study $74 \%$ of study subjects showed normal blood pressure response to standing and $26 \%$ had borderline response. In control subjects also $60 \%$ showed normal and $40 \%$ had borderline response. In the present study $50 \%$ of somatoform disorder patients had normal response to isometric hand grip test (IHG) whereas $26 \%$ and $24 \%$ had borderline and abnormal result respectively. Comparing study subjects with control subjects no significant difference for mean score of blood pressure response to standing or IHG test response was found to exist indicating no change in sympathetic activity among somatoform disorder patients compared to control subjects.

So, from this study finding it is evident that sympathetic dysregulation may not have any role in the pathogenesis of somatoform disorders though these patients had reduced parasympathetic activity. Reduced parasympathetic activity indicates low vagal tone or autonomic rigidity which may lead to many cardiovascular disturbances.

\section{CONCLUSIONS}

Somatoform disorder patients had reduced parasympathetic activity which was significantly different from control subjects. However, no significant difference was present in sympathetic activity between somatoform disorder subjects and control population. According to ICD-10, individual who presents with repeated physical symptoms, together with persistent requests for medical investigations, in spite of repeated negative findings and reassurances by doctors that these symptoms have no physical basis, are categorized as somatoform disorders. ${ }^{17}$ However this does not mean that these symptoms are without physiological correlates. Although there may not be any evidence of discrete organic pathology, demonstrable physiological changes can still increase the risk of development and maintenance of physical complaints. ${ }^{18}$ In the most general sense, there is empirical evidence of a link between somatoform disorders and altered functioning of central nervous system which has gradually replaced suggested abnormalities in specific organ system. ${ }^{19}$ These study findings serve to remind us that somatoform disorders are not to be regarded as "all in mind." 20 This disease should get more attention from treatment perspectives as this lowered parasympathetic activity may result in catastrophic health problem. As the sample size was small, further study is needed with larger sample size.

\section{REFERENCES}

[1] Kirmayer LJ, Paillefer S. Somatoform disorders. In: Turner SM, Hersen M, eds. Adult psychopathology and diagnosis. $3^{\text {rd }}$ edn. New York: Wiley 1997: p. 333-83.

[2] De Wall MW, Arnold IA, Eekhof JH, et al. Somatoform disorders in general practice: prevalence, functional impairment and comorbidity with anxiety and depressive disorders. British Journal of Psychiatry 2004;184(6):470-6.

[3] Kumar R, Yeragani VK. Psyche and soma: new insights into the connection. Indian Journal of Psychiatry 2010;52(Suppl 1):S233-9. 
[4] Sharpe M, Peveler R, Mayou R. The psychological treatment of patients with functional somatic symptoms: a practical guide. Journal of Psychosomatic Research 1992;36(6):515-29.

[5] Brown RJ. Psychological mechanisms of medically unexplained symptoms: an integrative conceptual model. Psychological Bulletin 2004;130(5):793-812.

[6] Ewing DJ, Clarke BF. Diagnosis and management of diabetic autonomic neuropathy. Br Med J (Clin Res Ed) 1982;285(6346):916-8.

[7] The 10th revision of International Statistical Classification of Diseases and Related Health Problems (ICD-10). Geneva: World Health Organization, 1992.

[8] Basu S, Dasgupta SK. Adaptation of GHQ - 28 into Bengali. Department of Applied Physiology. Kolkata: University of Calcutta. (PhD Dissertation - 1996).

[9] Misra UK, Kalita J. Clinical Neurophysiology. $3^{\text {rd }}$ edn. New Delhi: Elsevier India Pvt. Ltd., 2014.

[10] Moore M, Brown D, Money N, et al. Mind-body skills for regulating the autonomic nervous system Defense Centers of Excellence for psychological health and traumatic brain injury. 2011: Version 2. http://www.compmed.umm.edu /http://www.wholistichealingresearch.com

[11] Deveci A, Taskin O, Dinc G, et al. Prevalence of pseudoneurologic conversion disorder in an urban community in Manisa, Turkey. Social Psychiatry and Psychiatric Epidemiology 2007;42(11):857-64.
[12] Wise MG, Rundell JR. Clinical Manual of Psychosomatic medicine: a guide to consultation-liaison psychiatry. Washington D.C.: American Psychiatric Publishing 2005: p. 121-49.

[13] Hall JE. Guyton and Hall Textbook of Medical Physiology. 12th edn. India: Saunders an imprint of Elsevier 2011: p. 729-40.

[14] American Psychiatric Association (APA). Diagnostic and statistical manual of mental disorders. $4^{\text {th }}$ edn Washington D.C.: APA 1994.

[15] Izci F, Hocagil H, Izci S, et al. P-wave and QT dispersion in patients with conversion disorder. The Clin Risk Manag 2015;11:475-80.

[16] Zimmermann-Viehoff F, Thayer J, Bergt J, et al. Herat rate variability during in patient psychosomatic treatment - a naturalistic observational study. Z Psychosom Med Psychother 2016;62(1):20-31.

[17] Berntson GG, Cacioppo JT, Quigley KS. Cardiac psychophysiology and autonomic space in humans: Empirical perspectives and conceptual implications. Psychol Bull 1993;114(2):296-322.

[18] Rief W, Barsky AJ. Psychobiological perspectives on somatoform disorders. Psychoneuroendocrinology 2005;30(10):996-1002.

[19] Wessely S, Nimnuan C, Sharpe M. Functional somatic syndromes: one or many? Lancet 1999;354(9182):936-9.

[20] White PD, Moorey S. Psychosomatic illnesses are not "all in mind". J Psychosom Res 1997;42(4):329-32. 